\title{
LOCALLY CONVEX TOPOLOGICAL LATTICES
}

BY

\author{
ALBERT R, STRALKA
}

\begin{abstract}
The main theorem of this paper is: Suppose that $L$ is a topological lattice of finite breadth $n$. Then $L$ can be embedded in a product of $n$ compact chains if and only if $L$ is locally convex and distributive. With this result it is then shown that the concepts of metrizability and separability are equivalent for locally convex, connected, distributive topological lattices of finite breadth.
\end{abstract}

In [8] R. P. Dilworth proved that every distributive lattice of finite breadth $n$ could be embedded (algebraically) in a product of $n$ chains. Since finite breadth and distributivity are hereditary properties this result served to characterize distributive lattices of finite breadth. Dyer and Shields in [9] and Anderson in [4] (also see question 90 of [7]) asked if a result of a similar nature could be obtained for topological lattices, specifically: Can every compact, connected, metric, distributive topological lattice of breadth $n$ be embedded in an $n$-cell? This question was answered affirmatively and more generally by Kirby A. Baker and the present author in [6]. For easier reference this result along with several consequences appears as Theorem 1.3 below.

The major result of this paper is more nearly the topological analogue to Dilworth's theorem. We characterize the class of those topological lattices which can be embedded in a finite product of compact chains as the class of locally convex, distributive topological lattices of finite breadth. The class of locally convex topological lattices is rather large. For example, it contains all compact topological lattices [15], all locally compact and connected topological lattices [3], and all discrete lattices. This being the case our result contains those of [6] and [8].

We also show that the set of separating points of a locally convex, connected topological lattice is very well behaved. This fact together with our main theorem allows us to prove that separability and metrizability are equivalent for locally convex, connected, distributive topological lattices of finite breadth.

The author wishes to express his gratitude to F. Burton Jones for his aid in the preparation of this paper.

1. Definitions and preliminary results. A topological lattice is a Hausdorff topological space with a pair of continuous maps $\wedge, \vee: L \times L \rightarrow L$ such that

Received by the editors January 10, 1970.

AMS 1969 Subject Classifications. Primary 5456, 0665; Secondary 5453.

Key Words and Phrases. Topological lattice, locally convex, compact, connected, separating point, embed, product of compact chains, distributive.

Copyright (C) 1970, American Mathematical Society 
$(L, \wedge, \vee)$ is a lattice. Now, let $L$ be such a lattice. $A \subseteq L$ is meet-redundant if there is a proper subset $B$ of $A$ such that $\bigwedge A=\bigwedge B$. A set which is not meetredundant is meet-irredundant. We say that $L$ has breadth $n$, written $\operatorname{Br}(L)=n$, if $L$ has a meet-irredundant subset consisting of $n$ elements, but every subset of $L$ with $n+1$ elements is meet-redundant. $A$ is said to be a representation of $x_{0}$ if $x_{0}=\bigwedge A$. Let $x_{0} \in L$ define $\rho\left(x_{0}\right)$ to be the largest cardinality of those meetirredundant representations of $x_{0}$. For $A \subseteq L, \rho(A)=\max \{\rho(a) ; a \in A\}$. Note that $\rho(L)=\operatorname{Br}(L)$. For $a, b \in L$ with $a<b$ we define $[a, b]$ to be $\{x \in L ; a \leqq x \leqq b\}$. A subset $A$ of $L$ is convex if whenever it contains $\{a, b\}$ and $a \leqq b$ then it contains $[a, b] . L$ is locally convex if its topology has a base of convex sets.

For convenience we shall use the following notation.

$\mathscr{L}_{n}$ is the class of all locally convex, distributive topological lattices of finite breadth $n$.

$\mathscr{C}_{n}$ is the class of all compact, distributive topological lattices of finite breadth $n$.

By a chain we shall mean a linearly ordered set with a topology at least as large as the interval topology. It is obvious that every chain is a topological lattice. By $I$ we shall mean the real interval $[0,1]$ with its usual topology and its usual lattice operations. $I^{n}$ is the $n$-fold Cartesian product of $I$ and $\operatorname{Hom}(L, I)$ is the set of continuous homomorphisms of the lattice $L$ into $I$.

For connected chains the concepts of local convexity and "linearity" (in the sense of Jones [11]) are equivalent. Thus, Theorem 10 of [11] may be rephrased as follows.

THEOREM 1.1 (JONES [11]). Suppose that L is a member of $\mathscr{C}_{1}$ which is also connected and metric. Then $L$ is separable.

L. W. Anderson in [2] showed that locally convex, connected topological lattices are locally connected. With this fact we may modify Theorem 10 of [17] to read

THEOREM 1.2 (RoY [17]). Suppose that L is a locally convex, connected, metric topological lattice with no separating points such that every pair of points of $L$ can be separated by a closed separable subset of $L$. Then $L$ is separable.

The results of [6] which we mentioned in the introduction are now given as

THEOREM 1.3 ([6]). Let $L \in \mathscr{L}_{n}$. If $L$ is either (1) compact or (2) locally compact and connected, then $L$ can be embedded in a product of $n$ compact chains. Moreover if $L$ is also separable and metric then it can be embedded in $I^{n}$.

It should be pointed out that part (2) above is derived from part (1) via Corollary 3 of [5].

2. Some examples. We now give several examples which will be of use in the subsequent discussion. 
EXAMPLE 2.1. To form the lattice $L_{1}$ we take the real interval $[0,1]$ with its usual lattice operations and give it a new topology $\tau_{1} . \tau_{1}$ is that topology which is generated by the usual topology on $[0,1]$ and a "new" open set $Q$, the set of rational numbers in $[0,1] . L_{1}$ is a connected chain which is neither compact nor locally convex.

EXAMPLE 2.2. For each nonnegative integer let $c_{n}=1 / 2^{n}$. Let $A_{n}$ be the line segment from $\left(1, c_{n-1}\right)$ to $\left(0, c_{n}\right)$ and let $B_{n}$ be the line segment from $\left(0, c_{n}\right)$ to $\left(1, c_{n}\right)$ for each positive integer $n$. Define $L_{2}$ to be $\left(\bigcup_{n=1}^{\infty} A_{n}\right) \cup\left(\bigcup_{n=1}^{\infty} B_{n}\right) \cup\{(1,0)\}$. Give $L_{2}$ the topology which it inherits from the plane and let it have the separating point ordering with $\bigvee L_{2}=(1,1)$ and $\bigwedge L_{2}=(1,0)$. (I.e. $x<y$ if and only if the component of $L_{2} \mid\{x\}$ which contains $y$ also contains $(1,1)$.) $L_{2}$ is a connected chain which is neither locally convex nor compact.

EXAMPLE 2.3. Define $X$ to be the set of all points in the plane with nonnegative $y$-coordinates. We give $X$ the topology generated by the usual topology and all sets formed by taking the interiors of circles tangent to the $x$-axis along with the points of tangency. Let $C$ be the usual representation of the Cantor set on the interval from $(0,0)$ to $(1,0)$. Let $\left\{\left(a_{i}, b_{i}\right) ; i \in N\right\}$ be the set of pairs of $x$-coordinates of end points of complimentary intervals to $C$ with $a_{i}<b_{i}$. For example, $(1 / 2,2 / 3)$, $(1 / 9,2 / 9)$ and $(7 / 9,8 / 9)$ are in this set. Now form a set $\left\{x_{i} ; i \in N\right\}$ where $x_{i}$ is the mid-point of the interval $\left[a_{i}, b_{i}\right]$. Define $A_{i}$ to be the line segment from $\left(a_{i}, 0\right)$ to $\left(x_{i}, 1\right)$ and define $B_{i}$ to be the line segment from $\left(x_{i}, 1\right)$ to $\left(b_{i}, 0\right)$. Let $L_{3}=C \cup$ $\left(\bigcup_{i=1}^{\infty} A_{i}\right) \cup\left(\bigcup_{i=1}^{\infty} B_{i}\right)$. With the topology $L_{3}$ inherits from $X, L_{3}$ is a connected space. Give $L_{3}$ the separating point order with $\bigvee L_{3}=(1,0)$ and $\bigwedge L_{3}=(0,0)$. $L_{3}$ is a separable chain which is not locally convex and not metrizable.

EXAMPLES 2.4 AND 2.5. These examples are obtained from $L_{2}$ and $L_{3}$. We shall only describe $L_{2}$ since the description of $L_{3}$ is analogous. Replace each $A_{i}$ and $B_{i}$ in $L_{2}$ by connected, distributive two-dimensional topological lattices $A_{i}^{\prime}$ and $B_{i}^{\prime}$ so that $L_{4}=\left(\bigcup_{i=1}^{\infty} A_{i}^{\prime}\right) \cup\left(\bigcup_{i=1}^{\infty} B_{i}^{\prime}\right) \cup\{(1,0)\}$ is a cyclic chain in the sense of Whyburn [21] with $\left\{\left(x, c_{i}\right) ; i=1,2, \ldots\right.$ and $\left.x \in\{0,1\}\right\} \cup\{(1,0)\}$ being the set of separating points and end points.

EXAMPLE 2.6. Let $L_{6}$ be the set of nonnegative integers with the discrete topology. Define $1 \wedge x=0 \vee x=x, 0 \wedge x=0$ and $1 \vee x=1$ for all $x \in L_{6}$. For $x, y \in L_{6} \mid\{0,1\}$ define $x \wedge y=x$ if $x=y$ and 0 otherwise. Define $\vee$ dually. $L_{6}$ is a modular, nondistributive, locally convex topological lattice of breadth two. Moreover $L_{6}$ cannot be embedded in a compact topological lattice. This is seen by an elementary limit argument.

EXAMPLe 2.7. In the plane take $A$ to be the line segment from $(0,0)$ to $(1,0)$ and $B$ to be the line segment from $(0,1)$ to $(1,1)$. Define $L_{7}$ to be $A \cup B$ and give $L_{7}$ the lexicographic order. Then with the interval topology $L_{7}$ is a locally convex, separable chain which is not metrizable.

3. The main theorem. The goal of this section is to prove our main result which is 
THEOREM 3.1. Suppose that $L$ is a topological lattice of finite breadth $n$. Then $L$ can be embedded (algebraically and topologically) in a product of $n$ compact chains if and only if $L$ is locally convex and distributive.

It is apparent that the chains mentioned above can be taken to be connected. While the proof of the sufficiency is obvious we will need a sequence of lemmas to establish the necessity.

LEMMA 3.1. Let $L$ be a distributive topological lattice of finite breadth. Then Hom $(L, I)$ separates points in $L$.

Proof. We use essentially the same proof that is used in [18]. If $L$ is a chain then since the topology on $L$ contains the interval topology, $L$ is normally ordered in the sense of Nachbin [15]. Then upon applying Theorem 1 of Chapter 2 of [15] and Lemma 2 of [5] our proof is complete. Note that connectivity is not required in the last mentioned reference.

The author is indebted to the referee for the next observation.

Proposition 3.1. Let $L \in \mathscr{L}_{n}$. If $0 \in L$ then $L$ has a basis of convex sublattices at 0 .

Proof. Let $U$ be a neighborhood of 0 . Let $V$ be a convex neighborhood of 0 in $V$. From the continuity of $V$ there is a neighborhood $W$ of 0 such that $V^{n} W \subseteq v$. $W \wedge L$ is then the desired neighborhood.

The proof of Theorem 3.1 depends upon the embedding theorem of S. Mrówka [14]. The next definition and Lemma 3.3 recast certain concepts used in [14] into terms more appropriate for the present situation.

Definition. Let $L$ be a topological lattice. The set $\left\{\left(f_{i}, F_{i}\right) ; i=1, \ldots, m\right\}$ is said to *-separate the point $p$ of $L$ and the closed subset $K$ of $L$ if $m$ is finite and each $f_{i}$ is a continuous homomorphism of $L$ into a compact, distributive topological lattice $F_{i}$ such that one, and hence both, of the following two equivalent conditions holds.

(i) $f_{1} \times \cdots \times f_{n}(p) \notin f_{1} \times \cdots \times f_{m}(K)^{*}$,

(ii) $K$ can be written as $K_{1} \cup \cdots \cup K_{m}$ where for each $i, f_{i}(p) \notin f_{i}\left(K_{i}\right)^{*}$.

The proof that these two conditions are equivalent will be omitted since it is straightforward.

LeMma 3.2. Suppose that $L \in \mathscr{L}_{n}$ satisfies the property that every point $p$ and every closed subset $K$ of $L$ not containing $p$ can be *-separated. Then $L$ can be embedded in a product of $n$ compact chains.

Proof. Suppose that $\mathscr{F}=\left\{\left(f_{\alpha}, F_{\alpha}\right) ; \alpha \in \Gamma\right\}$ is the class of all pairs $\left(f_{\alpha}, F_{\alpha}\right)$ where $F_{\alpha}$ is a compact, distributive topological lattice and $f_{\alpha}$ is a continuous homomorphism of $L$ into $F_{\alpha}$. From Lemma 3.1, F separates the points of $L$ and hence is nonempty. Define $f: L \rightarrow \times_{\alpha \in \Gamma} F_{\alpha}$ by $f(x)_{\alpha}=f_{\alpha}(x)$. With the usual algebraic and topological structure $X_{\alpha \in \Gamma} F_{\alpha}$ is a compact topological lattice and since $\mathscr{F}$ separates 
points $f$ is a continuous monomorphism. Since every point $p$ and every closed set $K$ not containing $p$ can be *-separated, it follows from Mrówka's embedding theorem [14] that $f$ is an iseomorphism. $f(L)^{*}$ is a compact, distributive topological lattice and by a simple limit argument it can be shown that $\operatorname{Br}\left(\varphi(L)^{*}\right)=\operatorname{Br}(L)$. By Theorem 1.3 above we may conclude that $\varphi(L)^{*}$ and hence $L$ can be embedded in a product of $n$ compact chains.

Theorem 3.1 will be proved by induction on breadth. The next lemma will be the first step in the induction argument.

Lemma 3.3. Suppose that $L \in \mathscr{L}_{1}$. Then $L$ can be embedded in a compact chain (i.e., a member of $\mathscr{C}_{1}$ ).

Proof. Suppose that $p \in L$ and $K$ is a closed subset of $L$ which does not contain $p$. Since $L$ is linearly ordered $K=K_{0} \cup K_{1}$ where $K_{0}=(p \wedge L) \cap K$ and $K_{1}=(p \vee L)$ $\cap K$. No generality is lost by assuming that $K_{0} \neq \varnothing \neq K_{1}$. If there is $q \in L$ such that $p<q \leqq k$ for every $k \in K_{1}$, choose $\varphi_{1} \in \operatorname{Hom}(L, I)$ so that $\varphi_{1}(p)=1 / 2$ and $\varphi_{1}(q)=1$. (Such a map exists by Lemma 3.1.) If no such point $q$ exists define $\varphi_{1}: L \rightarrow I$ by $\varphi_{1}(p \wedge L)=1 / 2$ and $\varphi_{1}\left(\left(K_{1} \vee L\right)^{*}\right)=1$. Thus in either situation there is a homomorphism $\varphi_{1}$ of $L$ into $I$ such that $\varphi_{1}(p)=1 / 2$ and $\varphi_{1}\left(K_{1}\right)=1$. In a similar fashion one can construct a homomorphism $\varphi_{0}$ of $L$ into $I$ such that $\varphi\left(K_{0}\right)=0$ and $\varphi(p)=1 / 2$. Combining these two homomorphisms in the appropriate way we obtain a homomorphism $\varphi: L \rightarrow I$ such that $\varphi(p) \notin \varphi(K)^{*}$. Thus by Lemma 3.2 $L$ can be embedded in a compact chain.

The next lemma is the key result in our discussion.

Lemma 3.4. Suppose that Theorem 3.1 holds for every lattice in $\bigcup_{m=1}^{n-1} \mathscr{L}_{m}$. Let $L \in \mathscr{L}_{n}$ and suppose that $L$ has a minimum element 0 . If $K$ is a closed subset of $L$ which does not contain 0 then 0 and $K$ can be ${ }^{*}$-separated.

Proof. First we show that we may assume that $K=(K \vee L)^{*}$. Suppose that such an assumption cannot be made. This implies that there are nets $\left\{k_{\alpha} ; \alpha \in \Gamma\right\}$ and $\left\{x_{\alpha} ; \alpha \in \Gamma\right\}$ in $K$ and $L$ respectively such that the net $\left\{k_{\alpha} \vee x_{\alpha} ; \alpha \in \Gamma\right\}$ converges to 0 . Since $L$ is locally convex and since $k_{\alpha} \vee x_{\alpha} \geqq k_{\alpha} \geqq 0$ for each $\alpha \in \Gamma$ the net $\left\{k_{\alpha} ; \alpha \in \Gamma\right\}$ must converge to 0 . However this is not possible because $K$ is closed and $0 \notin K$. Hence we shall assume that $K=(K \vee L)^{*}$.

From Proposition 3.1 there is a neighborhood $W$ of 0 which is a sublattice of $L$ such that $W \cap K=\varnothing$. Note that since $W$ is convex and $0 \in W$ it follows that $W$ is an ideal of $L$. Choose $x_{0} \in W$ so that $\rho\left(x_{0}\right)=\rho(W)$. We may assume that $x_{0}=0$, for otherwise, since $0 \notin K$ we could map $L$ onto $x_{0} \vee L$ and proceed with the same proof. If $\rho(0)=1$ then $W$ must be linearly ordered. If $W \neq\{0\}$ choose $q \in W \backslash\{0\}$ and choose $\varphi \in \operatorname{Hom}(L, I)$ so that $\varphi(0) \neq \varphi(q)$. If $W=\{0\}$ then define $\varphi: L \rightarrow I$ by $\varphi(0)=0$ and $\varphi(L \mid\{0\})=1$. In both situations we have succeeded in defining a continuous homomorphism of $L$ into $I$ such that $\varphi(0) \notin \varphi(K)^{*}$. Thus 0 and $K$ can be *-separated. 
Now suppose that $\rho(0)=m>1$. Let $\left\{x_{1}, \ldots, x_{m}\right\}$ be a meet-irredundant representation of 0 . Define a set $\left\{y_{1}, \ldots, y_{m}\right\}$ by letting $y_{i}=\bigwedge_{j \neq i} x_{j}$. It follows readily that $\left\{y_{1}, \ldots, y_{m}\right\}$ is join-irredundant (see the proof of Theorem 1 of [4]) and $y_{i} \wedge y_{j}=0$ if and only if $i \neq j$.

One of the following two cases must then hold.

Case (i). Suppose that the set $\left\{x_{1}, \ldots, x_{m}\right\}$ can be chosen so that $\left\{y_{1}, \ldots, y_{m}\right\} \subseteq W$. Define a map $\theta: L \rightarrow X_{i=1}^{m}\left[0, y_{i}\right]$ by $\theta(t)=\left(t \wedge y_{1}, \ldots, t \wedge y_{m}\right) . \theta$ is a continuous homomorphism. We now claim that $\theta(0) \notin \theta(K)^{*}$. Suppose, on the contrary, that $\theta(0) \in \theta(K)^{*}$. This implies that there is a net $\left\{k_{\alpha} ; \alpha \in \Gamma\right\}$ of members of $K$ such that the net $\left\{k_{\alpha} \wedge y_{i} ; \alpha \in \Gamma\right\}$ converges to 0 for $i=1, \ldots, m$. For $\alpha \in \Gamma$ we propose to show that some element of the set $\left\{y_{1}, \ldots, y_{m}, k_{\alpha}\right\}$ is join-redundant. Suppose that this is not so. Form a set $\left\{z_{1}, \ldots, z_{m+1}\right\}$ by defining $z_{i}=\left(\bigvee_{i \neq j} y_{j}\right) \vee k_{\alpha}$ for $1 \leqq i \leqq m$ and $z_{m+1}=\bigvee_{i=1}^{m} y_{i} \cdot\left\{z_{1}, \ldots, z_{m+1}\right\}$ is meet-irredundant and

$$
z_{1} \wedge \cdots \wedge z_{m+1}=\left(z_{1} \wedge \cdots \wedge z_{m}\right) \wedge\left(y_{1} \vee \cdots \vee y_{m}\right)
$$

$W$ is a sublattice of $L$ and $\left\{y_{1}, \ldots, y_{m}\right\} \subseteq W$ so we must have $y_{1} \vee \cdots \vee y_{m+1} \in W$. Then since $W$ is also an ideal of $L$ by (1) above $z_{1} \wedge \cdots \wedge z_{m+1} \in W$. Thus we have found an element of $W, z_{1} \wedge \cdots \wedge z_{m+1}$, such that $\rho\left(z_{1} \wedge \cdots \wedge z_{m+1}\right)=m+1>\rho(W)$. This is not possible because of the way in which $\rho$ was defined so $\left\{y_{1}, \ldots, y_{m}, k_{\alpha}\right\}$ must be join-redundant. If $k_{\alpha}$ were redundant in this set then we would have $k_{\alpha} \leqq y_{1} \vee \cdots \vee y_{m}$, i.e., $k_{\alpha} \in W$. But of course this is not possible since $W \cap K=\varnothing$. Then because $m$ is finite we may assume that $y_{1}$ is join-redundant in $\left\{y_{1}, \ldots, y_{m}, k_{\alpha}\right\}$ for each $\alpha \in \Gamma$. This implies that

$$
\begin{aligned}
y_{1} & =y_{1} \wedge\left(y_{2} \vee \cdots \vee y_{m} \vee k_{\alpha}\right) \\
& =\left(y_{1} \wedge y_{2}\right) \vee \cdots \vee\left(y_{1} \wedge y_{m}\right) \vee\left(y_{1} \wedge k_{\alpha}\right) \\
& =0 \vee\left(y_{1} \wedge k_{\alpha}\right)=y_{1} \wedge k_{\alpha} .
\end{aligned}
$$

However we than have

$$
y_{1}=\lim y_{1}=\lim \left(y_{1} \wedge k_{\alpha}\right)=0
$$

which contradicts the fact that $y_{1} \neq 0$. Thus $\theta(0) \notin \theta(K)^{*}$.

$\operatorname{Br}\left(\left[0, y_{i}\right]\right)<\operatorname{Br}(L)$ by Lemma 1 of [5]. Hence by hypothesis $\left[0, y_{i}\right]$ can be embedded in a compact topological lattice. Then since $\theta(0) \notin \theta(K)^{*}$ there is a continuous homomorphism $\psi$ of $L$ into a compact topological lattice such that $\psi(0) \notin \psi(K)^{*}$. Thus in this case 0 and $K$ can be *-separated. We now turn to the alternative situation.

Case (ii). Suppose that it is not possible to choose $\left\{x_{1}, \ldots, x_{m}\right\}$ so that $\left\{y_{1}, \ldots, y_{m}\right\}$ $\subseteq W$. We may choose $\left\{x_{1}, \ldots, x_{m}\right\}$ so that the number of elements of $\left\{y_{1}, \ldots, y_{m}\right\}$ which are in $W$ is maximum. Also we may assume that $y_{1} \notin W$. Suppose that there is an element $y \in\left(\left[0, y_{1}\right] \backslash\left\{0, y_{1}\right\}\right) \cap W$. Define $\left\{y_{1}^{\prime}, \ldots, y_{m}^{\prime}\right\}$ and $\left\{x_{1}^{\prime}, \ldots, x_{m}^{\prime}\right\}$ as follows: $y_{1}^{\prime}=y, y_{i}^{\prime}=y_{i}$ for $i \neq 1$ and $x_{i}^{\prime}=\bigvee_{j \neq i} y_{j}^{\prime}$ for each $i$. By elementary calculation it is easily shown that 
(a) $\left\{x_{1}^{\prime}, \ldots, x_{m}^{\prime}\right\}$ is meet-irredundant.

(b) $\bigwedge_{i=1}^{m} x_{i}^{\prime}=0$.

(c) The set of $y$ 's corresponding to $\left\{x_{1}^{\prime}, \ldots, x_{m}^{\prime}\right\}$ is $\left\{y_{1}^{\prime}, \ldots, y_{m}^{\prime}\right\}$.

This leads us to the conclusion that the number of elements in $\left\{y_{1}^{\prime}, \ldots, y_{m}^{\prime}\right\} \cap W$ is larger by one than the number of elements in $\left\{y_{1}, \ldots, y_{m}\right\} \cap W$. This is not possible so we must have $\left[0, y_{1}\right] \cap W=\{0\} . K$ may be written as the union of two closed sets $K_{0}$ and $K_{1}$ where $K_{0}=\left\{k \in K ; y_{1} \wedge k=0\right\}$ and $K_{1}=\left\{k \in K ; y_{1} \wedge k \in(L \backslash W)^{*}\right\}$. $\operatorname{Br}\left(\left[0, y_{1}\right]\right)<\operatorname{Br}(L)$ and $0 \notin\left(y_{1} \wedge K_{1}\right)^{*}$, so by hypothesis there is a continuous homomorphism $\tau$ of $L$ into a compact topological lattice $X$ such that $\tau(0) \notin \tau\left(K_{1}\right)^{*}$. Now let $A=\left\{x \in L ; y_{1} \wedge x=0\right\}$. From Lemma 1 of [5], $\operatorname{Br}(A)<n=\operatorname{Br}(L)$. Note that $K_{0}$ is a closed subset of $A$ which does not contain 0 . By hypothesis and Lemma 3.2, $A$ can be embedded in a product of $r=\operatorname{Br}(A)$ compact chains $C_{1} \times \cdots \times C_{r}$. For $i=1, \ldots, r$ let $\varphi_{i}$ be the map formed by composing the embedding map of $A$ into $C_{1} \times \cdots \times C_{r}$ with the natural projection of $C_{1} \times \cdots \times C_{r}$ onto $C_{i}$. From this it follows that $K_{0}$ may be written as the union of $r$ sets $M_{1} \cup \cdots \cup M_{r}$ where $\varphi_{i}(0) \notin \varphi_{i}\left(M_{i}\right)^{*}$ (in $C_{i}$ ). Choose $m_{i} \in M_{i}$ for $i=1, \ldots, r$ and define $\sigma_{i}: L \rightarrow\left[0, m_{i}\right]$ by $\sigma_{i}(x)=m_{i} \wedge x$. We claim that $0 \notin \sigma_{i}\left(M_{i}\right)^{*}$. Suppose that $0 \in \sigma_{i}\left(M_{i}\right)^{*}$. Then there is a net $\left\{x_{\alpha} ; \alpha \in \Gamma\right\}$ of elements of $M_{i}$ such that the net $\left\{m_{\mathfrak{i}} \wedge x_{\alpha} ; \alpha \in \Gamma\right\}$ converges to 0 . This implies that the net $\left\{\varphi_{i}\left(m_{i} \wedge x_{\alpha}\right) ; \alpha \in \Gamma\right\}$ converges to $\varphi_{i}(0) \in C_{i}$. Since $C_{i}$ is a chain this leads to the conclusion that $\left\{\varphi_{i}\left(m_{i} \wedge x_{\alpha}\right) ; \alpha \in \Gamma\right\} \subseteq \varphi_{i}\left(M_{i}\right)$. However $\varphi_{i}(0) \notin \varphi_{i}\left(M_{i}\right)^{*}$. Thus we have a contradiction and our claim is valid. To complete our proof we note that then $\left\{(\tau, X),\left(\varphi_{1} \circ \sigma_{1}, C_{1}\right), \ldots,\left(\varphi_{k^{\circ} \sigma_{k}}, C_{k}\right)\right\}{ }^{*}$-separates 0 and $K$.

One more lemma is still needed before we can prove our theorem.

LEMMA 3.5. Suppose that $L$ is a locally convex topological lattice. Let $K$ be a closed subset of $L$ and let $p$ be a point in $L \backslash K$. Then there is a convex neighborhood $W$ of $p$ such that $K$ can be written as the union of two closed sets $K_{0}$ and $K_{1}$ with $\left(p \wedge K_{0}\right)$ $\cup\left(p \vee K_{1}\right) \subseteq L \backslash W$.

Proof. Suppose that our lemma is not true. Let $\mathscr{U}=\left\{U_{\alpha} ; \alpha \in \Gamma\right\}$ be a convex local base at $p$. There must be a net $\left\{k_{\alpha} ; \alpha \in \Gamma\right\}$ of elements of $K$ such that for each $\alpha \in \Gamma,\left\{p \wedge k_{\alpha}, p \vee k_{\alpha}\right\} \subseteq U_{\alpha}$. Thus $\lim p \vee k_{\alpha}=p=\lim p \wedge k_{\alpha}$. Since each $U_{\alpha}$ is convex and $p \wedge k_{\alpha} \leqq k_{\alpha} \leqq p \vee k_{\alpha}$ for each $\alpha \in \Gamma$, we must have lim $k_{\alpha}=p$. But $K$ is closed and $p \notin K$. Thus we have a contradiction. Hence our lemma is proved.

Finally, we may now complete the proof of our theorem.

Proof of Theorem 3.1. From Lemma 3.3 our theorem holds for lattices in $\mathscr{L}_{1}$. Assume that the theorem holds for lattices in $\bigcup_{m=1}^{n-1} \mathscr{L}_{m}$. Let $L \in \mathscr{L}_{n}$. Let $p \in L$ and let $K$ be a closed subset of $L$ such that $p \notin K$. Let $W, K_{0}$ and $K_{1}$ be those sets obtained by Lemma 3.5. It follows that $p \notin\left(p \wedge K_{0}\right)^{*} \cup\left(p \vee K_{1}\right)^{*}$. So by Lemma 3.4 there are continuous homomorphisms $f_{1}, \ldots, f_{k}$ of $L$ into compact lattices $F_{1}, \ldots, F_{k}$ such that

$$
f_{1} \times \cdots \times f_{k}(p) \notin f_{1} \times \cdots \times f_{k}\left(\left(p \vee K_{1}\right)^{*}\right)^{*}
$$


And from a dual formulation of Lemma 3.4 there are continuous homomorphisms $g_{1}, \ldots, g_{j}$ of $L$ into compact lattices $C_{1}, \ldots, C_{j}$ such that

$$
g_{1} \times \cdots \times g_{j}(p) \notin g_{1} \times \cdots \times g_{j}\left(\left(p \wedge K_{0}\right)^{*}\right)^{*} .
$$

Let $\sigma: L \rightarrow p \vee L$ be defined by $\sigma(x)=p \vee x$ and let $\mu: L \rightarrow p \wedge L$ be defined by $\mu(x)=p \wedge x$. It then follows that

$$
\left\{\left(f_{1^{\circ}} \sigma, F_{1}\right), \ldots,\left(f_{k^{\circ}} \sigma, F_{k}\right),\left(g_{1^{\circ}} \mu, \mathscr{C}_{1}\right), \ldots,\left(g_{j^{\circ}} \mu, \mathscr{C}_{j}\right)\right\}
$$

*-separates $p$ and $K$. Therefore, by Lemma 3.2, $L$ can be embedded in a product of $n$ compact chains.

In [12] J. D. Lawson gives an example of a compact, distributive topological lattice which has no continuous homomorphism onto $I$. Thus Theorem 3.1 cannot be extended to lattices of infinite breadth. Also Example 2.6 above shows that distributivity cannot be deleted from the list of hypotheses of Theorem 3.1. It should be noted that Theorem 3.1 answers partially but not completely the following two questions.

(1) Can every distributive topological lattice of finite breadth $n$ be embedded in a product of $n$ chains?

(2) Which topological lattices can be embedded in a compact topological lattice?

Our next theorem is the metric analog of Theorem 3.1.

THEOREM 3.2. Suppose that $L \in \mathscr{L}_{n}$ and suppose that $L$ is separable and metrizable. Then $L$ can be embedded in $I^{n}$.

Proof. From Theorem 3.1 there are homomorphisms $\varphi_{1}, \ldots, \varphi_{n}$ and compact chains $C_{1}, \ldots, C_{n}$ such that $\varphi_{1} \times \cdots \times \varphi_{n}$ embeds $L$ into $C_{1} \times \cdots \times C_{n}$. Let $D_{i}=$ $\varphi_{i}(L) . D_{i}$ is a separable metric chain for each $i$. We claim that $D_{i}$ can be embedded in $I$ for each $i$. Let $M_{i}=\{x \in L ; x \vee L$ is open in $L\}$. Since $D_{i}$ is separable and metric it satisfies the second axiom of countability. Hence $M_{i}$ is countable. Let $K_{i}$ be a countable dense subset of $D_{i} . M_{i} \cup K_{i}$ is then a countable order-dense subset of $D_{i}$. Thus from page 200 of [7] we can embed $D_{i}$ in $I$. It then follows easily that $L$ can be embedded in $I^{n}$.

Theorem 3.2 yields immediately the following corollary. This result complements the dimension-theoretic results which appear in [6] and [12].

COROLlary 3.2. Suppose that $L$ is a separable, metrizable member of $\mathscr{L}_{n}$. Then $\operatorname{Br}(L) \geqq \operatorname{dim} L$. Moreover, if $L$ is connected then $\operatorname{Br}(L)=\operatorname{dim} L$.

It is obvious what changes Theorem 5.1 and Theorem 5.2 below make in Theorem 3.2 and Corollary 3.2.

4. Separating points in locally convex, connected topological lattices. For a connected topological lattice $L$ we define a set $S(L)$ to be

$$
\{x \in L ; L=(x \vee L) \cup(x \wedge L)\} \text {. }
$$


$S(L)$ consists of the set of separating points of $L$ along with those universal bounds which $L$ might possess (see Theorem 1 of [2]). A point $x \in S(L)$ is a local maximum (minimum) if $x$ is an isolated point in $x \vee L(x \wedge L) . M(S(L))$ is the set of local maxima of $S(L)$ while $m(S(L))$ is the set of local minima. If $x \in m(S(L))$ and $y \in M(S(L))$ such that $x<y$ and $[x, y] \cap S(L)=\{x, y\}$, then $\langle x, y\rangle$ is said to be a gap and $x$ and $y$ are called adjacent gap points. If $C$ is a compact, metric chain then it is well known that $C$ can have at most a countable number of gaps. For example see Corollary 3.3 of [6].

Lemma 4.1. Suppose that $L$ is a locally convex, connected topological lattice. Then $S(L)$ is a closed linearly ordered sublattice of $L$ such that if $S_{0} \subseteq S(L)$ and $S_{0}$ is bounded above in $L$ then $\bigvee S_{0}$ exists and is in $S(L)$.

Proof. It is easily seen that $S(L)$ is a linearly ordered sublattice of $L$. Suppose that $\left\{s_{\alpha} ; \alpha \in \Gamma\right\}$ is a net in $S(L)$ converging to $s_{0}$. For $x \in L$ and $\alpha \in \Gamma$ either $x \leqq s_{\alpha}$ or $s_{0} \geqq x$. Hence $s_{0} \in S(L)$ and $S(L)$ is closed.

Suppose that $S_{0} \subseteq S(L)$ and suppose that $S_{0}$ has an upper bound $x_{0}$. If $\bigvee S_{0}$ exists and is in $S_{0}$ then we are done. So we suppose that if $\bigvee S_{0}$ exists then it is not in $S_{0}$, i.e. for each $s \in S$ there is $t \in S_{0}$ such that $s<t$. For $s \in S_{0}, L=A(s)$ $\cup B(s) \cup\{s\}$ where $A(s)=s \wedge L \mid\{s\}$ and $B(s)=s \vee L \mid\{s\}$. Let $A_{0}=\bigcup\left\{A(s) ; s \in S_{0}\right\}$ and let $B_{0}=\bigcap\left\{B(s) ; s \in S_{0}\right\}$. Since $x_{0} \in B(s)$, for every $s \in S_{0}, B_{0} \neq \varnothing$. Then because $S_{0} \subseteq A_{0}$ we have $L=A_{0} \cup B_{0} . A_{0}$ is open, $A_{0} \cap B_{0}=\varnothing$ and $L$ is connected so $A_{0}^{*} \cap B_{0} \neq \varnothing$. Choose $\left\{a_{\alpha} ; \alpha \in \Gamma\right\}$ a net of elements of $A_{0}$ converging to some point $s_{0} \in B_{0}$. For each $\alpha \in \Gamma$ there is $s_{\alpha} \in S_{0}$ such that $a_{\alpha} \leqq s_{\alpha} \leqq b_{0}$. Then since $L$ is locally convex there is a subnet of $\left\{s_{\alpha} ; \alpha \in \Gamma\right\}$ which converges to $s_{0}$. It then follows that $s_{0}=\bigvee S_{0}$ and since $S(L)$ is closed we have $s_{0} \in S(L)$.

The next theorem lists several properties of $S(L)$ when $L$ is locally convex. This result is similar to Theorem 2 of [20] in which A. D. Wallace proves that every compact, connected, metrizable topological lattice is a cyclic chain in the sense of Whyburn [21].

THEOREM 4.1. Suppose that $L$ is a locally convex, connected topological lattice. Then

(i) $S(L)$ is conditionally complete.

(ii) The topology which $S(L)$ inherits from $L$ coincides with the interval topology on $S(L)$.

(iii) If $a, b \in S(L)$ with $a<b$ then $[a, b] \cap S(L)$ is compact.

(iv) $S(L)$ is locally compact.

(v) If $L$ is metric then it possesses at most a countable number of gaps.

(vi) If $L$ is metric then $S(L)$ is separable.

Proof. Part (i) follows from Lemma 4.1 and p. 115 of [7]. Part (ii) is easily derived. Part (iii) follows from parts (i) and (ii) and p. 115 of [7]. Part (iv) follows directly from parts (ii) and (iii). 
Now let $S(L)$ be a metric space with metric $d$. Suppose that $S(L)$ has uncountably many gaps. This implies that $m(S(L))$ must also be uncountable. For each $x \in m(S(L))$ define $\zeta(x)$ to be a positive integer $n$ such that $B(x, 1 / n) \cap(x \vee L)=\{x\}$. $B(x, 1 / n)=\{y \in S(L) ; d(x, y)<1 / n\}$. Since $m(S(L))$ is uncountable there is a positive integer $n$ such that $\zeta^{-1}(n)$ is uncountable. We may assume that $n=1$. If both $\bigvee \zeta^{-1}(1)$ and $\wedge \zeta^{-1}(1)$ existed, then by part (iii) of this theorem

$$
\left[\bigwedge \zeta^{-1}(1), \bigvee \zeta^{-1}(1)\right] \cap S(L)
$$

would be a compact metric space. This would contradict our assumption that $\zeta^{-1}(1)$ is uncountable. Thus we may assume that $\bigvee \zeta^{-1}(1)$ does not exist. We may also assume that there is $x_{0} \in \zeta^{-1}(1)$ such that $\zeta^{-1}(1) \cap\left(x_{0} \vee S(L)\right)$ is uncountable. We define a function $f: \zeta^{-1}(1) \rightarrow \zeta^{-1}(1)$ by letting $f(x)$ be an element of $\zeta^{-1}(1)$ $\cap(x \vee S(L))$ such that $f(x) \notin B(x, 1)$. Since $\bigvee \zeta^{-1}(1)$ does not exist it follows that $f$ is well defined. Define an infinite sequence $\left\{x_{1}, x_{2}, x_{3}, \ldots\right\}$ in $\zeta^{-1}(1)$ by setting $x_{1}=x_{0}$ and letting $x_{n}=f^{n-1}\left(x_{0}\right)$ for $n>1$. This sequence is indeed infinite since $\zeta^{-1}(1) \cap\left(x_{0} \vee S(L)\right)$ is uncountable and each $\left[x_{n}, x_{n+1}\right]$ is compact. For the same reasons it follows that $\left\{x_{1}, x_{2}, x_{3}, \ldots\right\}$ is bounded above in $S(L)$. Let

$$
x=\bigvee\left\{x_{1}, x_{2}, x_{3}, \ldots\right\}
$$

Since $\left[x_{1}, x\right]$ is compact and metrix $x$ must be a limit point of $\left\{x_{1}, x_{2}, x_{3}, \ldots\right\}$. However this is not possible since $d\left(x, x_{n}\right)>1$ for each $n$. This is a contradiction. Thus $S(L)$ can have at most a countable number of gaps. Hence part (v) is proved.

Let $S_{1}=\{x \in S(L) ; x<y$ for all $y \in m(S(L)) \cup M(S(L))\}$ and let

$$
S_{2}=\{x \in S(L) ; x>y \text { for all } y \in m(S(L)) \cup M(S(L))\} .
$$

$S_{1}$ and $S_{2}$ are connected (if nonempty) locally convex, metric chains. Hence, by Theorem 1.1, $S_{1}$ and $S_{2}$ are separable if nonempty. From part (v) above the set of gaps in $S(L)$ is countable. Thus $m(S(L)) \cup M(S(L))$ is countable. Then $S(L) \mid S_{1} \cup S_{2}$ $=\bigcup_{i=1}^{\infty}\left\{\left[a_{i}, b_{i}\right] \cap S(L)\right\}$ where $a_{i}, b_{i} \in m(S(L)) \cup M(S(L))$ with $a_{i}<b_{i}$. Each $\left[a_{i}, b_{i}\right]$ $\cap S(L)$ is a compact metric space by part (iii) above. Hence $\left[a_{i}, b_{i}\right] \cap S(L)$ is separable for each $i$. It then follows that $S(L) \backslash S_{1} \cup S_{2}$ and hence $S(L)$ is separable.

The examples in $\S 2$, in particular Examples 2.4 and 2.5, show that local convexity is necessary in Lemma 4.1 and in Theorem 4.1.

5. Separability and metrizability in topological lattices. The relationship between separability and metrizability in connected topological spaces has been studied by Alexandroff, Jones, Roberts, Treybig and Roy, among others. See [1], [10], [16] and [19]. In this section we obtain results of a similar nature.

In one direction we have

THEOREM 5.1. Suppose that $L \in \mathscr{L}_{n}$ and suppose also that $L$ is separable and connected. Then $L$ is metrizable. Moreover $L$ can be embedded in $I^{n}$. 
Proof. Suppose that $K$ is a countable dense subset of $L$. From Theorem 3.1 we may consider $L$ to be a sublattice of $C=C_{1} \times \cdots \times C_{n}$ where each $C_{i}$ is a compact chain. Let $\pi_{i}$ be the natural projection of $C$ onto $C_{i}$ and let $D_{i}=\pi_{i}(L)^{*}$. If $a_{i}=\bigwedge D_{i}$ and $b_{i}=\bigvee D_{i}$, then $K_{i}=\pi_{i}(K) \cup\left\{a_{i}, b_{i}\right\}$ is a countable dense subset of $D_{i}$. Then it follows from connectivity that $\mathscr{B}=\left\{[a, b] \mid\{a, b\} ; a, b \in K_{i}\right.$ and $\left.a<b\right\}$ is a countable base for the topology of $D_{i}$. Hence by Urysohn's Theorem $D_{i}$ is metrizable. From Corollary 3.3 of [6], among other sources, $D_{i}$ can be embedded into $I$. We may then conclude that $L$ can be embedded in $I^{n}$.

Example 2.3 shows that local convexity is necessary in Theorem 3.1 while Example 2.7 shows that connectivity is necessary.

The next theorem is a partial converse to Theorem 5.1. The real interval $[0,1]$ with the discrete topology and the usual lattice operations is a simple example which shows that connectivity cannot be deleted from our next result.

THeOREM 5.2. Suppose that $L \in \mathscr{L}_{n}$ and suppose that $L$ is cornected and metric. Then $L$ is separable.

Proof. Our proof will be accomplished by means of induction on breadth. If $L$ satisfies the hypothesis of our theorem and $\operatorname{Br}(L)=1$, then, by Theorem $1.1, L$ is separable.

Now suppose that our theorem holds for lattices of breadth less than $n$ and suppose that $L$ is a lattice which satisfies the hypothesis above and $\operatorname{Br}(L)=n$. We consider two cases.

Case (i). Suppose that $L$ does not have any separating points. From Theorem 3.1, $L$ may be assumed to be a sublattice of $C=C_{1} \times \cdots \times C_{n}$ where each $C_{i}$ is a compact connected chain. Let $x=\left(x_{1}, \ldots, x_{n}\right)$ and $y=\left(y_{1}, \ldots, y_{n}\right)$ be two distinct points in $L$. There is some $i$ such that $x_{i} \neq y_{i}$. We may assume that $x_{i}<y_{i}$. Since $C_{i}$ is connected there is $c_{i} \in C_{\imath}$ such that $x_{i}<c_{i}<y_{i}$. Let $A=\left\{\left(a_{1}, \ldots, a_{n}\right) \in C ; a_{i}=c_{i}\right\} . A$ is a connected sublattice of $C$ with $\operatorname{Br}(A)=n-1$. It is easily seen that $A \cap L$ is a closed, locally convex, connected sublattice of $L$ such that $\operatorname{Br}(A \cap L) \leqq n-1$. By our induction hypothesis $A \cap L$ is separable. $A \cap L$ is then a closed separable subset of $L$ which separates $a$ and $b$. Thus the hypothesis of Theorem 1.2 is satisfied. Hence we may conclude that $L$ is separable.

Case (ii). Suppose that $L$ has separating points. $S(L)$ possesses those properties listed in Theorem 4.1. Let $K$ be a countable dense subset of $S(L)$. From Theorem 3.1 we can embed $L$ into a compact lattice. Let $a$ and $b$ be the universal bounds of $L$ in that lattice. The number of gaps in $S(L)$ is at most countable so it follows that the number of gaps in $S(L) \cup\{a, b\}$ is at most countable. Let $\left\{\left\langle a_{i}, b_{i}\right\rangle ; i \in N\right\}$ be the set of gaps of $S(L) \cup\{a, b\}$. For each $i,\left[a_{i}, b_{i}\right]$ satisfies the conditions of Case (i). Thus $\left[a_{i}, b_{i}\right]$ has a countable dense subset, say $K_{i}$. The set $\left(\bigcup_{i=1}^{\infty} K_{i}\right) \cup K$ is a countable dense subset of $L$. Thus $L$ is separable.

In the previous theorem distributivity was only used in Case (i). There it was used to show that if $L$ is a distributive lattice with breadth $n$ and $a$ and $b$ are distinct 
points of $L$ then there is a sublattice $L_{0}$ of $L$ such that $L_{0}$ separates $a$ and $b$ in $L$ and $\operatorname{Br}\left(L_{0}\right)<n$. Whether a wider class of lattices than distributive lattices share this property is unknown to the author. For such lattices Theorem 5.2 will hold.

\section{BIBLIOGRAPHY}

1. Paul Alexandroff, Über die metrization der im kleinen kompakten topologischen Räume, Math. Ann. 94 (1924), 294-301.

2. L. W. Anderson, On the distributivity and simple connectivity of plane topological lattices, Trans. Amer. Math. Soc. 91 (1959), 102-112. MR 21 \#1365.

3. - One dimensional topological lattices, Proc. Amer. Math. Soc. 10 (1959), 715-720. MR 21 \#6401.

4. - On the breadth and co-dimension of a topological lattice, Pacific J. Math. 9 (1959), 327-333. MR 21 \#4206.

5. - The existence of continuous lattice homomorphisms, J. London Math. Soc. 37 (1962), 60-62. MR 24 \#A3106.

6. K. A. Baker and A. R. Stralka, Compact, distributive lattices of finite breadth, Pacific J. Math. (to appear).

7. G. Birkhoff, Lattice theory, 3rd ed., Amer. Math. Soc. Colloq. Publ., vol. 25, Amer. Math. Soc., Providence, R. I., 1967. MR 37 \#2638.

8. R. P. Dilworth, A decomposition theorem for partially ordered sets, Ann. of Math. (2) 51 (1950), 161-166. MR 11, 309.

9. E. Dyer and A. Shields, Connectivity of topological lattices, Pacific J. Math. 9 (1959), 443-448. MR 21 \#4205.

10. F. B. Jones, A theorem concerning locally peripherally separable spaces, Bull. Amer. Math. Soc. 41 (1935), 437-439.

11. - Concerning certain linear abstract spaces and simple continuous curves, Bull. Amer. Math. Soc. 45 (1939), 623-628. MR 1, 45.

12. J. D. Lawson, Lattices with no interval homomorphisms, Pacific J. Math. 32 (1970), 459-466.

13. - The relation of breadth and codimension in topological semilattices. II (to appear).

14. S. Mrówka, Further results on E-compact spaces. I, Acta Math. 120 (1968), 161-185. MR 37 \#2165.

15. L. Nachbin, Topology and order, Van Nostrand, Princeton, N. J., 1956. MR 36 \#2125.

16. J. H. Roberts, A problem of Treybig concerning separable spaces, Duke Math. J. 28 (1961), 153-155. MR 24 \#A1705.

17. Prabir Roy, Separability of metric spaces, 149 (1970), 19-43.

18. E. D. Shirley and A. R. Stralka, Homomorphisms on connected topological lattices, Duke Math. J. (to appear).

19. L. B. Treybig, Concerning certain locally peripherally separable spaces, Pacific J. Math. 10 (1960), 697-704. MR 22 \#7104.

20. A. D. Wallace, Two theorems on topological lattices, Pacific J. Math. 7 (1957), 1239-1241. MR 20 \#824.

21. G. T. Whyburn, Analytic topology, Amer. Math. Soc. Colloq. Publ., vol. 28, Amer. Math. Soc., Providence, R. I., 1942. MR 4, 86.

UNIVERSITy OF CALIFORNIA,

RIVERSIDE, CALIFORNIA 92502 